\title{
A Remark on a Theorem of Powers and Sakai
}

\author{
S. Doplicher \\ Istituto Matematico "G. Castelnuovo", Università di Roma, I-00185 Roma, Italy
}

\begin{abstract}
Given an abelian locally compact group $G$ and a $C^{*}$-algebra with unit $\mathfrak{A}$, the set of those continuous representations of $G$ by automorphisms of $\mathfrak{A}$ which fulfill a spectrum condition is closed.
\end{abstract}

In a recent paper [1] Powers and Sakai proved, among other things, that if a sequence of continuous one-parameter groups of automorphisms of a $C^{*}$-algebra with identy, each with a generator in the algebra, converges strongly, uniformly on compact sets of the line, the limit one parameter group has a ground state.

As any one parameter group with a generator in the algebra has a ground state [1, proof of Theorem 2.3, first paragraph] this theorem is implied by a closedness property of the set of one parameter groups having a ground state.

The purpose of this note is to remark that from the algebraic spectrum condition [2] this closedness property follows naturally for any locally compact abelian group replacing the line.

Let $G$ be a locally compact abelian group and $\mathfrak{U}$ a $C^{*}$-algebra with identity $I$; let $\mathscr{A}$ be the set of all continuous homomorphisms of $G$ into the group of $*$ automorphisms of $\mathfrak{A}$, equipped with the strong topology.

For $\alpha \in \mathscr{A}$, by a representation of $\{\mathfrak{U}, \alpha\}$ we mean a covariant representation $(\pi, U): \pi$ is a representation of $\mathfrak{A}$ on a Hilbert space $\mathscr{H}$ and $U$ a strongly continuous unitary representation of $G$ on $\mathscr{H}$ s.t. $U(g) \pi(\cdot) U(g)^{-1}=\pi \circ \alpha_{g}, g \in G$.

If $\omega$ is an $\alpha$-invariant state on $\mathfrak{A},\left(\pi_{\omega}, U_{\omega}\right)$ and $\xi_{\omega}$ denote respectively the G.N.S. covariant representation and the associated cyclic vector s.t. $\omega=\left(\xi_{\omega}, \pi_{\omega}(\cdot) \xi_{\omega}\right)$ and $U_{\omega}(g) \pi_{\omega}(A) \xi_{\omega}=\pi_{\omega}\left(\alpha_{g}(A)\right) \xi_{\omega} ; g \in G, A \in \mathfrak{U}$. of $\hat{G}$.

Let $\hat{G}$ denote the dual group of $G$ and $K \subset \hat{G}$ a closed set including the identity

Let $\mathfrak{I}(\alpha, K)$ denote the smallest left ideal in $\mathfrak{U}$ including the set:

$\mathfrak{B}(\alpha, K)=\left\{\alpha_{f}(A) / A \in \mathfrak{U} ; \quad f \in L^{1}(G), \quad \hat{f} \mid K=0\right\}$,

where $\alpha_{f}(A)=\int f(g) \alpha_{g}(A) d \mu(g)$ and $\mu$ is a Haar measure on $G$.

The following conditions on $\alpha \in \mathscr{A}$ are equivalent:

(i) there exists an $\alpha$-invariant state $\omega$ on $\mathfrak{A}$ with spectrum $U_{\omega} \subset K$;

(ii) $\mathfrak{I}(\alpha, K) \neq \mathfrak{U}$. 
In Ref. [2] this is proved for $G=\mathbb{R}^{4}$ and $K=$ the future light cone, but that argument has a straightforward generalization to our present case.

Let $\mathscr{A}(K)$ be the set of $\alpha \in \mathscr{A}$ fulfilling (i)/(ii).

1. Proposition. The set $\mathscr{A}(K)$ is closed in $\mathscr{A}$ for the topology of strong convergence uniformly on compact sets of $G$. The set $\mathscr{A}(K)$ is sequentially closed in $\mathscr{A}$ for the topology of simple strong convergence.

Proof. If $\alpha \in \mathscr{A} \backslash \mathscr{A}(K)$ we have $\mathfrak{I}(\alpha, K)=\mathfrak{A}$ i.e.

$$
I=\sum_{i=1}^{N} A_{i} \alpha_{f_{i}}\left(B_{i}\right)
$$

with $f_{i} \in L^{1}(G), \hat{f}_{i}=0$ on $K$ and $A_{i}, B_{i} \in \mathfrak{A}, i=1, \ldots, N$. Given $\varepsilon>0$ let $C$ be a compact set in $G$ and $\varphi_{1}, \ldots, \varphi_{N}$ continuous functions with support in $C$ s.t.

$$
\left\|\varphi_{i}-f_{i}\right\|_{1}<\varepsilon, \quad i=1, \ldots, N \text {. }
$$

Let $\mathscr{N}(\alpha)$ be the compact-strong neighbourhood of $\alpha$ in $\mathscr{A}$ defined by

$$
\alpha^{\prime} \in \mathscr{N}(\alpha) \text { if } \sup \left\{\left\|\alpha_{g}^{\prime}\left(B_{i}\right)-\alpha_{g}\left(B_{i}\right)\right\| / g \in C, i=1, \ldots, N\right\}<\varepsilon \text {. }
$$

If $\alpha^{\prime} \in \mathscr{N}(\alpha), \sum_{i=1}^{N} A_{i} \alpha_{f_{i}}^{\prime}\left(B_{i}\right) \equiv B^{\prime} \in \mathfrak{I}\left(\alpha^{\prime}, K\right)$ and

$$
\begin{aligned}
\left\|I-B^{\prime}\right\| & \leqq \sum_{i=1}^{N}\left\|A_{i}\right\| \cdot\left\|\left(\alpha_{f_{i}}-\alpha_{f_{i}}^{\prime}\right)\left(B_{i}\right)\right\| \\
& \leqq \varepsilon \cdot \sum_{i=1}^{N}\left\|A_{i}\right\|\left(2\left\|B_{i}\right\|+\left\|\varphi_{i}\right\|_{1}\right) ;
\end{aligned}
$$

for small $\varepsilon, B^{\prime}$ is regular in $\mathfrak{U}$ and $\mathfrak{I}\left(\alpha^{\prime}, K\right)=\mathfrak{A}$; so the last equation holds for all $\alpha^{\prime}$ in a compact-strong neighbourhood of $\alpha$ in $\mathscr{A}$.

Let $\left\{\alpha^{(n)}\right\} \subset \mathscr{A}$ be a sequence and $\alpha \in \mathscr{A}$ s.t. for any fixed $g \in G$ and $A \in \mathfrak{A}$

$$
\left\|\alpha_{g}^{(n)}(A)-\alpha_{g}(A)\right\| \rightarrow 0 \quad \text { as } n \rightarrow \infty \text {. }
$$

For each $A \in \mathfrak{U}$ and $f \in L^{1}(G)$, we have that

$$
\left\|\left(\alpha_{f}^{(n)}-\alpha_{f}\right)(A)\right\| \leqq \int|f(g)| \cdot\left\|\alpha_{g}^{(n)}(A)-\alpha_{g}(A)\right\| d \mu(g)
$$

and $|f(g)| \cdot\left\|\alpha_{g}^{(n)}(A)-\alpha_{g}(A)\right\| \leqq 2\|A\| \cdot|f(g)|$; by Lebesgue theorem

$$
\left(\alpha_{f}^{(n)}-\alpha_{f}\right)(A) \rightarrow 0 \quad \text { as } n \rightarrow \infty \text {. }
$$

Assume $\alpha^{(n)} \in \mathscr{A}(K)$ but (1) holds. Setting $B^{(n)}=\sum_{i=1}^{N} A_{i} \alpha_{f_{i}}^{(n)}\left(B_{i}\right)$, by (1) and (2)

$$
B^{(n)} \rightarrow I \quad \text { as } n \rightarrow \infty \text {; }
$$

since $B^{(n)} \in \mathfrak{I}\left(\alpha^{(n)}, K\right) \neq \mathfrak{A}$ for all $n,(3)$ cannot hold, and also $\mathfrak{I}(\alpha, K) \neq \mathfrak{A} \|$.

2. Theorem. Let $\left\{\alpha^{(n)}\right\} \subset \mathscr{A}$ be a sequence s.t. for each $g \in G ; A \in \mathfrak{A} ; \alpha_{g}^{(n)}(A)$ is convergent in $\mathfrak{U}$; then the limit $\alpha_{g}(A)$ defines on element $\alpha \in \mathscr{A}$. If $\alpha^{(n)} \in \mathscr{A}(K)$, also $\alpha \in \mathscr{A}(K)$.

Proof. The limit $\alpha_{g}$ of $\alpha_{g}^{(n)}$ defines clearly a homomorphism of $G$ into the group of *automorphisms of $\mathfrak{A}$; by Proposition 1 we need only to prove that if $A \in \mathfrak{U}, g \in G \rightarrow \alpha_{g}(A)$ is continuous. Since $g \in G \rightarrow \alpha_{g}^{(n)}(A)$ is continuous it is also locally strongly measurable and so is $g \in G \rightarrow \alpha_{0}(A)$ by [3, Theorem 3.5.4]. Then continuity follows from local strong measurability by a known generalization of [3, Theorem 10.2.3].

Acknowledgements. I wish to thank Professor Sakai for sending his work before publication. 


\section{References}

1. Powers, R.T., Sakai, S.: Existence of ground states and KMS states for approximately inner dynamics. Commun. math. Phys. (to appear)

2. Doplicher, S.: An algebraic spectrum condition. Commun. math. Phys. 1, 1 (1965)

3. Hille,E., Phillips, R.S.: Functional analysis and semigroups. Providence, R.I.: Amer. Math. Soc. colloquim. publ. 1957

Communicated by H. Araki

(Received January 21, 1975; in revised form May 20, 1975) 
